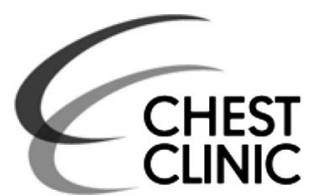
CLHEST

\title{
Cochrane corner: psychological interventions for individuals with cystic fibrosis and their families
}

\author{
Lutz Goldbeck, ${ }^{1}$ Astrid Fidika, ${ }^{1}$ Marion Herle, ${ }^{1}$ Alexandra L Quittner ${ }^{2}$
}

${ }^{1}$ Department of Child and Adolescent Psychiatry/

Psychotherapy, Medical Centre, University of Ulm, Ulm,

Germany

${ }^{2}$ Department of Psychology, University of Miami, Miami, Florida, USA

\section{Correspondence to} Lutz Goldbeck, Department of Child and Adolescent Psychiatry/Psychotherapy, Medical Centre, University of Ulm, Krankenhausweg 3, 89075 Ulm, Germany; lutz.goldbeck@uniklinik-ulm.de

This article is based on a Cochrane Review published in the Cochrane Database of Systematic Reviews 2014, Issue 6. Art. No.: CD003148. DOI: $10.1002 / 14651858$

CD003148.pub3. The Cochrane Database of Systematic Reviews should be consulted for the most recent version of the review, because Cochrane Reviews are updated regularly.

Received 14 May 2015 Revised 29 May 2015 Accepted 31 May 2015 Published Online First 19 June 2015

\begin{abstract}
Psychological issues associated with cystic fibrosis may arise from the patients' lifelong disease- and treatmentrelated burden. This Cochrane Review aimed to determine psychosocial and physical outcomes of psychological interventions. Trial registries, databases and professional networks were used to identify relevant studies. Altogether, 16 studies involving 556 participants were included. They were heterogeneous in their methods, design, target groups, and outcomes. Overall, the current evidence for psychological interventions is insufficient. Preliminary evidence was available for interventions targeting specific aspects of the treatment regimen, such as behavioural nutrition interventions.
\end{abstract}

\section{INTRODUCTION}

The growing population of individuals with cystic fibrosis (CF) face various psychological issues as a consequence of the disease burden and the complex treatment regimen. Research on psychological distress and adjustment to the disease in patients with $\mathrm{CF}$ indicates that most of the patients adjust well. Nevertheless, recent research highlights that symptoms of depression and anxiety are prevalent in particular subgroups of patients and their caregivers. ${ }^{1}$ These findings have led to an ongoing discussion about how to identify and address potential comorbid mental health issues in clinical practice. ${ }^{23}$

The negative impact of psychological distress on adherence to treatment and disease outcomes are also outlined in the literature. Thus, psychological interventions for $\mathrm{CF}$ may aim to improve mental health and psychological function, but adherence to treatment and medical outcomes are additional challenges that need to be addressed.

In this review, we have updated a prior review to provide current evidence for psychological interventions for individuals with $\mathrm{CF}$ and their families.

\section{METHODS}

Randomised controlled trials (RCTs) and quasi-randomised controlled studies evaluating psychological interventions were included provided they met the following inclusion criteria:

- Psychological interventions based on psychotherapeutic or psychosomatic techniques were applied.

- Interventions were facilitated by a psychologist or trained and supervised professional.

To cite: Goldbeck $L$, Fidika A, Herle $M$, et al. Thorax 2015;70:

1089-1091.
- They were conducted in an individual, family or group setting.

Educational interventions to promote adherence were not included. All interventions were classified into the following types of interventions: cognitivebehavioural, cognitive, family systems or systemic, psychodynamic and other interventions. Well-being and psychological functioning, pulmonary function and weight and height or body mass index were predefined as primary outcomes. The search strategies were updated and the most recent search was run in December 2013. Three authors were involved in the screening of abstracts and checking full texts for eligibility. Data were extracted by two authors. All data were analysed using a random-effects model. Results were pooled when more than one study examined the same type of intervention, or when the intervention was comparable in their methods, setting, outcomes, participants and control condition.

More detailed information about the methods being used can be found in the original Cochrane Review. ${ }^{4}$

\section{RESULTS}

\section{Included studies}

The search revealed a total of 1705 titles and abstracts. Of those, 33 new studies were examined for eligibility, with eight of these studies included. Altogether, 16 studies with 556 participants were included in the current version of the review. Furthermore, 22 studies are awaiting classification and four studies were classified as ongoing. Participants were individuals with $\mathrm{CF}$ of all age groups (early childhood to adulthood), as well as their caregivers. Most of the studies (12) were conducted in the USA. A wide range of psychological interventions were evaluated and various designs and methods were used. Overall, the quality of studies was heterogeneous and just one study was judged to have low risk of bias across all criteria.

\section{Main effects of psychological interventions}

The main findings are reported in this short version and details of those are demonstrated in table 1 . More detailed findings for each comparison of the studies in the review can be found in the original Cochrane Review. ${ }^{4}$

Five studies applying cognitive-behavioural interventions aimed to improve adherence and one study aimed to improve psychosocial adjustment. The single study addressing psychosocial adjustment used an intervention based on problem solving and developing social skills in a sample of children 


\begin{tabular}{ll} 
Psychological intervention/comparison & Outcomes \\
\hline 1) Cognitive behavioural & Secondary: \\
'Nutritional intervention plus behavioural & $\begin{array}{l}\text { Total calories consumed per } \\
\text { management training compared with }\end{array}$ \\
$\begin{array}{l}\text { nutritional intervention alone' } \\
\text { (children aged 4-12 years, group setting) }\end{array}$ & $\begin{array}{l}\text { post-intervention } \\
\text { Change in calorie intake } \\
\text { pre and post treatment }\end{array}$ \\
& $\begin{array}{l}\text { Percentage of estimated energy } \\
\text { requirements (\%EER) } \\
\text { post intervention } \\
\text { Change in \%EER } \\
\text { pre and post treatment }\end{array}$ \\
\end{tabular}

\section{2) Cognitive}

'Decision aid for patients considering lung transplantation compared with usual care (patients with advanced CF considering referral for lung transplantation, individual setting)

\section{3) Other interventions}

'Biofeedback-assisted breathing re-training compared with biofeedback-assisted relaxation training'

(individuals with CF; 10-41 years; individual setting)

\section{4) Other interventions}

'Massage therapy compared with bedtime reading control'

(children and adolescents with CF aged 5-18 years; individual setting)

\section{Secondary:}

Participants' knowledge

four-item questionnaire

(range 0-4)

3-week follow-up change in knowledge

week 3-baseline

Patient expectations

two-item questionnaire (range

0-2) 3-week follow-up

Patient expectations-change

in expectation score

week 3-baseline

Decisional conflict-total score range 0-100 (low decisional conflict to high decisional conflict)

Primary:

Pulmonary function-

$\mathrm{FEV}_{1}$ expressed in litres

\section{Pulmonary function - forced expiratory flow $25 \%-75 \%$ expressed in litres per second Primary: \\ Pulmonary function- peak expiratory flow rate Follow-up day 30 \\ Parent anxiety-State Trait Anxiety Inventory (STAl; range 0-80; higher scores=more anxiety) Child anxiety- \\ State Trait Anxiety Inventory for Children (STAIC; ranges from 0 to 80 with a higher score reflecting more anxiety)}

Participants' knowledge3-week follow-up
Assumed risk-control group(s)

The mean ranged across control groups from 1316 to 2315 calories.

The mean ranged across control groups from 303.9 to 489 calories

The mean in the control group was $127 \%$

The mean of change in the control group was $27 \%$

The mean score of participants' knowledge in the control group was 1.974

The mean in the control group was 0.3

The mean in the control group was 0.58

The mean in the control group was 0.05

The mean in the control group was 20.4

The mean in the control group was 0.78

The mean in the control group was 1.39

The mean in the control group was 244

The mean score in the control group was 40

The mean child anxiety score in the control group was 32.9
Corresponding risk-intervention group(s)

The mean in the intervention group was 0.54 higher (0.15-0.93 higher)

The mean in the intervention group was 0.67 higher (0.1-1.24 higher)

The mean in the intervention groups was 275.8 calories higher (66.65-485.05 higher)

The mean in the intervention groups was $\mathbf{3 6 4 . 0 6}$ calories higher (191.99-536.13 higher)

The mean in the intervention group was $21 \%$ higher $(7.76-34.24$ higher)

The mean of change in the intervention group was $21 \%$ higher (9.22-32.78 higher)

The mean score of participants knowledge in the intervention group was 0.98 higher (0.66-1.31 higher)

The mean in the intervention group was 0.94 higher (0.53-1.35 higher)

The mean in the intervention group was 0.73 higher (0.51-0.95 higher)

The mean in the intervention group was 0.66 higher (0.37-0.95 higher)

The mean in the intervention group was 8.8 lower (13.7-3.9 lower)

Number of participants (studies)

82 (3 studies)

82 (3 studies)

67 (1 study)

67 (1 study)

149 (1 study)

49 (1 study)

149 (1 study)

149 (1 study)

149 (1 study)

24 (1 study)

26 (1 study)

The mean in the intervention group

20 (1 study) was $\mathbf{5 3 . 9}$ higher (43.27 lower to 151.07 higher)

The mean in the intervention group was 9.1 lower (17.84-0.36 lower)

20 (1 study)

The mean child anxiety

score in the intervention

group was 8.2 lower (12.36-4.04 lower)

The basis for the on a Cochrane Review published by Goldbeck et al. comparison group and the relative effect of the intervention (and its $95 \% \mathrm{CI}$ ).

$\mathrm{CF}$, cystic fibrosis.

with CF (8-12 years). The five studies applying cognitivebehavioural techniques used educational and cognitivebehavioural methods to improve the nutritional status of underweight children (4-12 years). Data of three of the five studies could be pooled for the variables 'total calories consumed per day', and data of two studies for 'change in weight' to evaluate the effects of behavioural management plus nutrition education versus nutrition education alone. Underweight children receiving the additional behavioural component consumed more calories per day at post-intervention and showed significantly more increase in calorie intake compared with the group receiving nutrition education alone. No significant differences were found for the primary outcome 'change in weight'. Furthermore, one study of those five demonstrated significant differences between groups, with the combined intervention leading to higher achieved percentage of the individual estimated energy requirements (\%EER) at post-intervention and greater increases in prepost $\%$ EER. 
Interventions in three studies used cognitive-only techniques. One of these studies aimed to improve adherence via motivational interviewing and one via a written self-disclosure intervention. The third study provided an evidence-based decision aid for patients being considered for lung transplantation. In this study, patients aged 18 years or older using this structured decision aid reported significant improvements of their knowledge of their options and their realistic expectations of risk of surgery at 3 weeks of follow-up. These improvements were significantly greater in the intervention group compared with the control group. Furthermore, patients receiving the cognitive intervention reported lower decisional conflict scores 3 weeks later. Group differences for change scores were not significant.

One family systems study was included, which evaluated an intervention providing support to parents and children within the CF-community. No study using a psychodynamic approach met the inclusion criteria.

Moreover, six studies providing other types of interventions, namely self-hypnosis, biofeedback, massage therapy, music therapy, telemedicine intervention to support patients awaiting transplantation and dance/movement therapy were included. In one study, biofeedback-assisted breathing re-training was compared with biofeedback-assisted relaxation training. Adolescent and adult patients using the biofeedback-assisted breathing re-training had significantly better lung function measured with $\mathrm{FEV}_{1}$ expressed in litres and forced expiratory flow 25\%-75\% than the relaxation control group. One study evaluated massage therapy against bedtime reading for children and adolescents. Massage therapy provided by parental caregivers was superior to bedtime reading in reducing parents' anxiety and children's anxiety at 30 days of follow-up.

An overall effect of psychological interventions could not be obtained within this review due to the heterogeneous interventions and outcomes.

\section{DISCUSSION}

Limited evidence of psychological interventions for children with CF and their caregivers is reported in this systematic review, though some of the included studies showed promising findings. There is substantial evidence supporting cognitivebehavioural interventions combined with education to improve calorie intake in children. Preliminary evidence was found for an intervention combining an educational session with a standardised decision-making aid, as well for interventions involving biofeedback, massage and music therapy.

While there is some evidence for interventions targeting specific aspects of the treatment regimen, the overall evidence on psychological interventions for individuals with CF and their families is insufficient. Important psychological outcomes, such as adherence to inhalation treatments, or comorbid symptoms of depression or anxiety, have not been targeted so far. The primary limitations of research were the lack of randomised controlled studies and the diversity of interventions used of the studies meeting inclusion criteria. Although a wide range of psychological methods, target groups, stages of the disease and outcomes have been examined, most findings are based on single studies with small samples which may limit the reliability of these results. Only cognitive-behavioural interventions have demonstrated an effect in larger samples across multiple studies.

Overall, the quality of study methods was poor. Authors' reporting of information about study protocols was particularly inadequate. Although the most recent studies paid more rigorous attention to standards of intervention research and reporting, there is still room for improvement. CONSORT standards, which are available as well for non-pharmacological trials, ${ }^{5}$ should be consulted.

For future investigations, we recommend to investigate studies addressing issues that have not yet been targeted, such as parents' adjustment to diagnosis, patients' adherence to treatment, transition to adult care, comorbid depression and anxiety, and palliative care. Replication of findings in larger multicentre studies is necessary to consolidate and extend the preliminary findings of studies in this review. New technological approaches, such as internet-based psychological interventions, should be considered to overcome the barriers for conducting large trials in an orphan disease.

With regard to clinical practices, implementation of interventions as clinical routine requires identification of individuals in need of psychological or psychotherapeutic support. The availability of professionals who are located in CF teams or who train members of the team to provide interventions must also be addressed. The results of the international epidemiological data on symptoms of anxiety and depression ${ }^{1}$ initiated an open discussion about sense and necessity of routine screenings in CF care. $^{2} 3$ The International Guidelines Committee on Mental Health for Patients with CF and Their Caregivers is currently preparing recommendations on how to identify and treat patients and caregivers in need. Although specific intervention studies in patients with CF and comorbid mental disorders are missing, evidence-based treatments for anxiety and/or depression, such as exposure-based cognitive-behavioural therapy (CBT) for anxiety, or CBT aiming at activation and cognitive restructuring for depression should be considered for those patients and caregivers and are likely to improve their well-being and their adaptation to treatment.

Acknowledgements We are very grateful for the support and guidance of the Cochrane Cystic Fibrosis and Genetic Disorders Group. We would also like to thank the Neurosis, Anxiety and Depression Group from the Cochrane Collaboration for their help with the search strategy.

Contributors LG takes principal responsibility for updating the review. Screening titles, abstracts, reviewing papers and appraising quality of papers were done by $L G$, $\mathrm{AF}$ and $\mathrm{MH}$. Data were extracted and entered into RevMan by AF and MH. AF took the leading role in contacting authors of papers for additional information. She investigated data analysis, data management for the review and provided advice on conducting the update of the systematic review. All authors were involved in interpretation of the results of data analysis. ALQ provided general advice on the review and interpretation of results for the current update. She has contributed a broad overview to the process of updating this review. ALQ initiated the debate surrounding evidence-based interventions with the international psychosocial interest group, which enhanced the discussion of the findings. LG and AF drafted the updated review. All authors approved the final manuscript.

Competing interests The authors $L G$ and $A L Q$ are investigators of some of the considered interventions studies for this review.

Provenance and peer review Not commissioned; internally peer reviewed.

\section{REFERENCES}

1 Quittner AL, Goldbeck L, Abbott J, et al. Prevalence of depression and anxiety in patients with cystic fibrosis and parent caregivers: results of The International Depression Epidemiological Study across nine countries. Thorax 2014:69:1090-7.

2 Webb AK, Bryon M. The International Depression Epidemiological Study (TIDES): unfinished business? Thorax 2014:69:1067-8.

3 Quittner AL, Barker D, Goldbeck L, et al. Letter to the editor: authors' response. Thorax 2015;70:490-1.

4 Goldbeck L, Fidika A, Herle M, et al. Psychological interventions for individuals with cystic fibrosis and their families. Cochrane Database Syst Rev 2014;(6):CD003148.

5 Boutron I, Moher D, Altman DG, et al. Extending the CONSORT statement to randomized trials of nonpharmacologic treatment: explanation and elaboration. Ann Intern Med 2008;148:295-309. 